\title{
SCREENING FOR HEAT TOLERANCE IN SPRING WHEAT (TRITICUM AESTIVUM L.)
}

\author{
AA Khan*, AKM Shamsuddin, NCD Barma, MK Alam and MA Alam \\ Wheat Research Centre, Bangladesh Agricultural Research Institute, Gazipur-1701, Bangladesh
}

Accepted: $12^{\text {th }}$ September 2014

\begin{abstract}
The present investigation was undertaken to identify the promising heat tolerant lines and to evaluate their heat stress responses. Twenty five spring wheat genotypes were studied in non-stressed (optimum sowing) and stressed (late sowing) environments. The experiments were conducted at Regional Wheat Research Centre, Bangladesh Agricultural Research Institute, Gazipur, during the cropping season of 2009-10. Randomized complete block design was used with three replications. Yield and yield contributing phenological and physiological characters varied among the genotypes under both optimum and late sowing conditions. Ground cover, grain filling duration, canopy temperature at grain filling and biomass production were severely affected by the heat stress leading to low grain yield under late sowing condition. G-12, G-13, G-14, G-18, and G-19 were identified as heat tolerant genotypes based on their relative performance in yield components, grain yield and heat susceptibility indices. Present genotypes are found to be ideal candidates to be used in developing heat tolerant wheat varieties.
\end{abstract}

Key words: Heat tolerance, Heat stress susceptibility index, Spring wheat

\section{INTRODUCTION}

Wheat (Triticum aestivum L.) ranks second among major cereals next to rice and plays a vital role in food security of teeming hungry millions of Bangladesh. In the coming period leading up to 2020, demand of wheat for human consumption in developing countries is expected to grow at $1.6 \%$ per annum (Ortiz et al. 2008). Thus yield increase is very much essential to maintain global food security. Recent researches on climate change predict marked increases in both rainfall and temperature. The temperature is projected to rise by as much as $3-4{ }^{0} \mathrm{C}$ by the end of the century in South Asia (DEFRA 2005). Therefore, heat stress has been given the top research priority in major wheat growing regions, in particular in the developing world including Bangladesh (CIMMYT 1995). In the rice-wheat cropping system of Bangladesh, $80-85 \%$ of wheat is grown after harvesting of transplanted aman rice, of which about $60 \%$ are late planted due to delayed harvesting of rice (Barma et al. 2011). Late planted wheat often encounters high temperature stress during late March to mid April at grain filling period causing sig-

\footnotetext{
*Corresponding author: aakhan_so@yahoo.com
}

nificant yield reduction. There is a potential yield decline ( $1.3 \%$ per day) when sown beyond optimum time $\left(30^{\text {th }}\right.$ November) in Bangladesh condition (Saunders 1988).

Heat tolerance is a complex phenomenon thus difficult to assess. Many selection criteria based on morpho-physiological traits were reported to be associated with performance under heat stress in wheat. Heat tolerant metabolism was reported to be indicated by longer leaf chlorophyll retention, canopy temperature depression, photosynthetic rate and leaf senescence (Rees et al. 1993, Rahman 1996, Reynolds et al. 1997 and Al-Khatib and Paulsen 1999). The other traits like biomass, 1000grain weight and grain yield are also highly sensitive to heat stress (Barma 2005; Rahman 2009). Tillering capacity, grain weight, spike fertility, spike number, grains per spike, early ground cover etc were also reported to be associated with yield under heat stress and hence heat tolerance (Acevedo et al. 1991; Kohli et al. 1991; Samad 1994; Mann,1994).

Genetic variation may exist within the wheat genotypes for heat tolerance, thus evaluation of 
local and exotic germplasm for heat tolerance is important. Xiyong et al. (2000) suggested that combination of both heat susceptibility index and geometric mean yield per plant (or kernel weight per spike) could be used as selection criteria for the evaluation of heat tolerant genotypes. Bruckner and Frohberg (1987) measured stress tolerances using stress susceptibility indices based on grain yield. Barma (2005) estimated heat stress susceptibility indices for 1000-grain weight and grain yield to differentiate overall heat tolerance of the genotypes. Taking the above mentioned views into account, the present study was aimed at identifying genotypes of wheat for heat tolerance.

\section{MATERIALS AND METHODS}

Twenty four spring wheat genotypes along with a popular heat tolerant variety 'Shatabdi' (as a check) were collected from the ongoing breeding program of Wheat Research Centre, Bangladesh Agricultural Research Institute, Dinajpur to establish two experiments during the cropping season of 2009-2010. The experiments were conducted at the experimental field of Regional Wheat Research Centre, Bangladesh Agricultural Research Institute, Joydebpur, Gazipur. Randomized complete block design was used with three replications. The experimental site was situated between $23^{\circ} 46^{\prime} \mathrm{N}$ latitude and $90^{\circ} 23^{\prime} \mathrm{E}$ longitude with elevation of $8 \mathrm{~m}$ above sea level. The climate of the area is characterized by wet summer and dry winter. Temperature data recorded during the concerned period is graphically presented in Fig. 1. Seeds of each genotype were sown in 6 lines $(20 \mathrm{~cm}$ apart) of plots of $2.5 \mathrm{~m}$ long. Standard agronomic practices were practiced during the crop growth. The central 1 metre of 4 rows of each plot were harvested for recording yield and primary yield contributing characters. Ground coverage was recorded visually at 35 days after sowing using $0-10$ scale. Chlorophyll content of leaves was measured in 5 fully expanded flag leaves in vivo by a Minolta SPAD metre at anthesis and 21 days after anthesis and expressed in spad unit. The canopy temperature was measured by a hand held infra-red thermometer twice at 3 days interval at vegetative and grain filling stage.

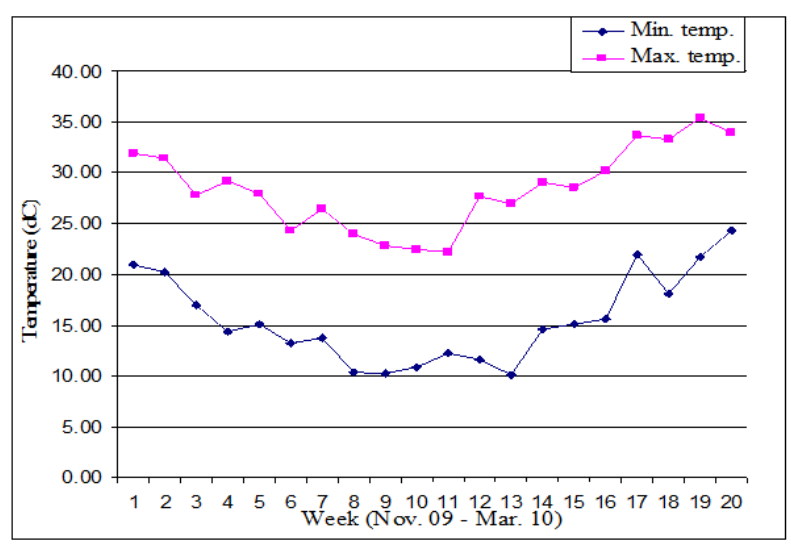

Fig. 1. Weekly average maximum and minimum temperatures at Regional Wheat Research Centre, Gazipur during the cropping season 2009-10

Analysis of variances was done for different characters according to the formula suggested by Steel and Torrie (1960). The ' $t$ ' values were calculated from the accession mean for each pair of characters under normal and late sowing following the formula used by Singh and Choudhary (1985). Heat stress susceptibility indices " $\mathrm{S}$ " were estimated for each character according to the equation as described by Fischer and Maurer (1978). The lower the "S" value the higher would be the level of tolerance to stress condition. The collected data were subjected to analysis of variance by using the statistical software MSTATC and mean separation was done by Duncan's Multiple Range Test (DMRT) (Gomez and Gomez 1984).

\section{RESULTS AND DISCUSSION}

\section{Performances of the genotypes in late sow- ing environment}

The genotypes differed significantly in phenological, physiological and primary yield contributing characters related to heat tolerance (Tables 1). The performances under late sowing condition (Tables 2) revealed pronounced effects of heat stress on the traits. In this study, heat stress resulted in early anthesis, senescence and physiological maturity, shortened grain filling duration, decreased chlorophyll content of flag leaf at 21 days after anthesis, 
1000-grain weight and biomass, reduced the number of spikes per unit area and grains per spike. Nevertheless, heat stress enhanced early ground cover and increased canopy temperature, chlorophyll content at anthesis and grain filling rate.

Genotypes G-2, G-3, G-4, G-12, G-13, G-14, G-17, G-18, G-19, G-21, G-24 and G-25 showed delayed anthesis suggesting their ability to withstand against heat stress. Al-Karaki (2012) reported that lengthening the preheading period of development would provide a better means of increasing grain yield under heat stress in durum wheat cultivars. Stay green of plants under stress conditions could be considered as an important trait since, longer stay green would be beneficial as it allows plants to retain their leaves actively for photosynthesis under stress condition (Koc et al. 2008). In this study, the genotypes G-1, G6, G-10, G-14, G-17, G-18, G-21 and G-24 delayed leaf senescence in late sowing condition, implying that these genotypes had the green foliage for longer period under heat stress condition. Similarly, the genotypes G-1, G-17 and G-18 showed delayed maturity in heat stress indicating their ability to stay green under heat stress. The genotypes G-1, G -5 , G-11, G-16, G-17 and G-18 took longer period for grain filling compared to those of the other genotypes under heat stressed condition. Paknejad et al. (2007) reported that the improvement of yield under drought stress has resulted from a more extended grain filling duration and a higher chlorophyll content.

Early ground cover was reported to be important in agronomic context (Rawson 1988; Badaruddin et al. 1999). High ground cover might reduce evaporative water loss from soil by providing better shade. Reynolds et al. (2001) stated that trait like ground cover could be important under heat stress. In wheat, ground cover probably a greater proportion of total evapotranspiration used in crop transpiration rather than soil evaporation. In this context, the genotypes G-1, G-3, G-5,
G-6, G-9, G-12, G-13, G-14, G-15, G-16, G17, G-18, G-19, G-20, G-21, G-22, G-24 and G-25 were found to have high ground coverage at mid vegetative stage under late sowing condition. Low canopy temperature and/or high canopy temperature depression have been used by several authors in screening for highly tolerant varieties to drought and heat (Amani et al. 1996; Ayeneh et al. 2002; Talebi 2011; Guendouz et al. 2012). Wheat genotypes with a low canopy temperature can maintain high transpiration and photosynthetic rate as well as produce a high yield under stressed conditions (Talebi 2011). Therefore, lower canopy temperature is regarded as an important mechanism of heat stress escape. In this study, the genotypes G-4, G-5, G-7, G8, G-9, G-10, G-11, G-12, G-13, G-14, G-15, G-17, G-18, G-19, G-20, G-21, G-22 and G24 showed low canopy temperature at vegetative stage suggesting that these genotypes can keep their canopy cool at vegetative stage under heat stressed condition. On the other hand, the genotypes G-4, G-7, G-9, G-15 and G-22 showed low canopy temperature at grain filling stage under late sowing condition suggesting their tolerance to high temperature at late sowing. Heat stress attributed to decline chlorophyll contents in cool-season cereal species leading to physiological changes and thereby leaf senescence (Almeselmani et al. 2011 and Dhyani et al. 2013). Therefore, higher retention of leaf chlorophyll under hot environment is often regarded as an expression of heat tolerance. Talebi (2011) confirmed chlorophyll content as a potential indicator for screening wheat genotypes for drought response. In this study, high chlorophyll contents of flag leaf at anthesis were found in the genotypes G-1, G2, G-4, G-5, G-6, G-7, G-8, G-10, G-11, G12, G-14, G-15, G-16, G-17, G-18, G-19, G20, G-21, G-22 and G-24 indicating their ability for the fixation of photosynthate under late sowing condition. Moreover, the genotypes $\mathrm{G}$ -5, G-6, G-7, G-10, G-11, G-13, G-14, G-15, G-16, G-17, G-18, G-19, G-20, G-21, G-22 and G-24 showed high chlorophyll content of flag leaf at 21days after anthesis under late 
sowing condition. Several authors (Blum et al. 1997; Khanna-Chopra and Viswanathan 1999; Singh et al. 2011) emphasized that selection for high biomass yield should bring about positive improvement in grain yield under drought and heat stress. Therefore, selection for biomass yield is one of the most important ways to improve the productivity under late sowing conditions. In our study, the genotypes G-1, G-2, G-5, G-6, G-14, G-17 and G-22 had produced high biomass under heat stressed condition. der late planting condition. Spikes number per unit area has been considered as a potential selection criteria for grain yield under heat stress (Reynolds et al. 1992; Hu and Rajaram 1994). In the present investigation, the genotypes G-2, G-5, G-9, G-10, G-13, G-17, G-19, G-22, G-23 and G-24 have produced higher number of spikes per square meter under late sowing condition. Grain weight is the most sensitive yield component to high temperature and could be used as a reliable trait to assess the responsiveness of genotypes to high tem-

Table 1 Analysis of variance (Mean square) for phenological, physiological and primary yield contributing characters in spring wheat under optimum and late sowing conditions

\begin{tabular}{|c|c|c|c|c|c|c|c|c|c|c|c|c|c|c|c|c|}
\hline \multirow[b]{2}{*}{$\begin{array}{l}\text { Sources } \\
\text { of } \\
\text { variation }\end{array}$} & \multirow[b]{2}{*}{ df } & \multicolumn{15}{|c|}{ Mean square } \\
\hline & & $\begin{array}{l}\text { Anthe- } \\
\text { sis } \\
\text { (day) }\end{array}$ & $\begin{array}{l}\text { FLS } \\
\text { (day) }\end{array}$ & $\begin{array}{l}\text { PM } \\
\text { (day) }\end{array}$ & $\begin{array}{l}\text { GFD } \\
\text { (day) }\end{array}$ & $\begin{array}{l}\mathrm{GC}_{35} \\
\text { (scale) }\end{array}$ & $\begin{array}{l}\mathrm{CT}_{\mathrm{vg}} \\
\left({ }^{\circ} \mathrm{C}\right)\end{array}$ & $\begin{array}{l}\mathrm{CT}_{\mathrm{gf}} \\
\left({ }^{\circ} \mathrm{C}\right)\end{array}$ & $\begin{array}{l}\mathrm{CHL}_{\mathrm{a}} \\
\text { (spad) }\end{array}$ & $\begin{array}{l}\mathrm{CHL}_{21} \\
\text { (spad) }\end{array}$ & $\begin{array}{l}\text { Biom }^{-2} \\
(\mathrm{~g})\end{array}$ & $\begin{array}{l}\text { GFR } \\
\left(\mathrm{gm}^{-2}\right. \\
\left.\mathrm{d}^{-1}\right)\end{array}$ & $\begin{array}{l}\text { Spikes } \\
\mathrm{m}^{-2} \\
\text { (no.) }\end{array}$ & $\begin{array}{l}\text { Grains } \\
\text { spike }^{-1} \\
\text { (no.) }\end{array}$ & $\begin{array}{l}1000- \\
\text { grain } \\
\text { wt. } \\
\text { (g) }\end{array}$ & $\begin{array}{l}\mathrm{GY} \\
\mathrm{m}^{-2} \\
(\mathrm{~g})\end{array}$ \\
\hline \multicolumn{17}{|c|}{ Optimum sowing condition } \\
\hline $\begin{array}{l}\text { Replica- } \\
\text { tion }\end{array}$ & 2 & 3.38 & 0.50 & 0.32 & 1.62 & 0.27 & 0.11 & 0.49 & 4.15 & 0.01 & 1058.00 & 0.26 & 144.50 & 31.05 & 25.95 & 18.00 \\
\hline $\begin{array}{l}\text { Geno- } \\
\text { type }\end{array}$ & 24 & $21.68 * *$ & $7.01 * *$ & $8.91^{* *}$ & $14.41 * *$ & $0.46^{* *}$ & $2.90^{* *}$ & $1.14 * *$ & $10.94 *$ & $12.00 * *$ & $29064.67 * *$ & $4.44 * *$ & $4759.37 * *$ & $103.25^{* *}$ & $43.59 * *$ & $6479.46^{* *}$ \\
\hline Error & 48 & 2.75 & 1.42 & 1.78 & 1.33 & 0.14 & 0.87 & 0.35 & 4.35 & 2.78 & 6618.41 & 1.33 & 1474.71 & 26.53 & 6.33 & 2183.62 \\
\hline \multicolumn{17}{|c|}{ Late sowing condition } \\
\hline $\begin{array}{l}\text { Replica- } \\
\text { tion }\end{array}$ & 2 & 0.18 & 0.02 & 0.32 & 0.02 & 0.19 & 0.19 & 0.08 & 9.95 & 0.08 & 288.00 & 2.62 & 722.00 & 15.79 & 0.30 & 1922.00 \\
\hline $\begin{array}{l}\text { Geno- } \\
\text { type }\end{array}$ & 24 & $4.28^{* *}$ & $4.74 * *$ & $4.83^{* *}$ & $5.58^{* *}$ & $0.45^{* *}$ & $1.49^{* *}$ & $1.60^{* *}$ & $6.43^{*}$ & $24.50 * *$ & $14154.71 * *$ & $5.32 * *$ & $2948.21 *$ & $91.31 * *$ & $31.73 * *$ & $3411.33^{* *}$ \\
\hline Error & 48 & 0.55 & 0.48 & 0.44 & 0.60 & 0.13 & 0.37 & 0.20 & 2.90 & 6.90 & 3941.12 & 1.68 & 1218.87 & 33.82 & 3.45 & 1234.50 \\
\hline
\end{tabular}

*and ** indicate significant at $5 \%$ and $1 \%$ levels of probability, respectively

$\mathrm{FLS}=$ Flag leaf senescence (day); $\mathrm{PM}=$ Physiological maturity (day); $\mathrm{GFD}=$ Grain filling duration (day); $\mathrm{GC}_{35}=$ Ground coverage at 35 days; $\mathrm{CT}_{\mathrm{vg}}=$ Canopy temperature at vegetative stage $\left({ }^{\circ} \mathrm{C}\right) ; \mathrm{CTgf}=\mathrm{Canopy}$ temperature at grain filling stage $\left({ }^{\circ} \mathrm{C}\right) ; \mathrm{CHL}_{\mathrm{a}}=$ Chlorophyll content at anthesis (spad unit); $\mathrm{CHL}_{21}=\mathrm{Chlorophyll}^{2}$ content at 21 days after anthesis ( spad unit); Bio $\mathrm{m}^{-2}=$ Biomass $\mathrm{m}^{-2}$; GFR= Grain filling rate $\left(\mathrm{g} \mathrm{m}^{-2} \mathrm{~d}^{-1}\right) ; \mathrm{GY}=$ Grain yield $\left(\mathrm{g} \mathrm{m}^{-2}\right) ; \mathrm{df}=$ Degrees of freedom

Grain filling rate in wheat influences grain yield under a wide range of conditions (Wheeler et al. 1996). Zahedi and Jenner (2003) reported that relatively higher grain filling rates could be taken as an important criterion for breeding genotypes acclimatized to late planting condition. The genotypes G-2, G-3, G-5, G-12, G-14, G-17, G-22, G-23 and G-24 showed high grain filling rates even un- perature (Sharma et al. 2008). The genotypes G-1, G-2 and G-13 showed higher 1000-grain weight under late sowing condition. However, the genotype G-16 has produced the highest number of grains per spike under late sowing condition. Grain yield is an important selection criteria for heat stress. Singh et al. (2011) stated that high grain yield of a genotype under late sowing condition indicates the pres- 
Table 2 Mean performances of the genotypes for phenological, physiological and primary yield contributing characters in spring wheat under late sowing condition

\begin{tabular}{|c|c|c|c|c|c|c|c|c|c|c|c|c|c|c|c|}
\hline $\begin{array}{l}\text { Geno- } \\
\text { type }\end{array}$ & $\begin{array}{l}\text { Anth } \\
\text {-esis } \\
\text { (day) }\end{array}$ & $\begin{array}{l}\text { FLS } \\
\text { (day) }\end{array}$ & $\begin{array}{l}\text { PM } \\
\text { (day) }\end{array}$ & $\begin{array}{l}\text { GFD } \\
\text { (day) }\end{array}$ & $\begin{array}{l}\mathrm{GC}_{35} \\
\text { (scale) }\end{array}$ & $\begin{array}{l}\text { CTvg } \\
\left({ }^{\circ} \mathrm{C}\right)\end{array}$ & $\begin{array}{l}\text { CTgf } \\
\left({ }^{\circ} \mathrm{C}\right)\end{array}$ & $\begin{array}{l}\text { CHLa } \\
\text { (spad ) }\end{array}$ & $\begin{array}{l}\mathrm{CHL}_{21} \\
\text { (spad) }\end{array}$ & $\begin{array}{l}\text { Bio } \mathrm{m}^{-2} \\
(\mathrm{~g})\end{array}$ & $\begin{array}{l}\text { GFR } \\
\left(\mathrm{g} \mathrm{m}^{-2}\right. \\
\left.\mathrm{d}^{-1}\right)\end{array}$ & $\begin{array}{l}\text { Spike } \\
\mathrm{m}^{-2} \\
\text { (no.) }\end{array}$ & $\begin{array}{l}\text { Grain } \\
\text { spike }^{-1} \\
\text { (no.) }\end{array}$ & $\begin{array}{l}1000- \\
\text { grain } \\
\text { wt.(g) }\end{array}$ & $\begin{array}{l}\text { Yield } \\
\mathrm{m}^{-2}(\mathrm{~g})\end{array}$ \\
\hline \multirow{2}{*}{$\begin{array}{l}\text { G 01 } \\
\text { (ck) }\end{array}$} & 65.5 & 91.5 & 96.0 & 30.5 & 5.75 & 26.25 & 33.20 & 49.2 & 41.5 & 1015.0 & 14.42 & 332.5 & 43.5 & 43.90 & 440.0 \\
\hline & b-e & $\mathrm{a}$ & $a b$ & $a b$ & $a b$ & $\mathrm{a}$ & $\mathrm{a}$ & a-e & d-h & a-e & b-g & b-e & $\mathrm{bc}$ & $a-c$ & $a b$ \\
\hline \multirow{2}{*}{ G 02} & 66.0 & 88.5 & 93.0 & 27.0 & 4.75 & 25.78 & 31.78 & 47.2 & 42.9 & 1017.5 & 16.48 & 365.0 & 50.4 & 45.70 & 445.0 \\
\hline & $a-d$ & $c-f$ & $c-f$ & $f-i$ & cd & $a b$ & $c-f$ & $a-e$ & $c-g$ & $a-d$ & $a b$ & $a-e$ & $\mathrm{bc}$ & & $a b$ \\
\hline \multirow{2}{*}{ G 03} & 66.5 & 88.5 & 93.0 & 26.5 & 5.50 & 25.35 & 31.50 & 45.4 & 36.2 & 902.5 & 15.13 & 317.5 & 50.3 & 38.00 & 400.0 \\
\hline & $a-c$ & $c-f$ & $c-f$ & $g-j$ & $a-c$ & $a-c$ & d-g & e & $\mathrm{h}$ & b-g & $a-e$ & $b-e$ & $\mathrm{bc}$ & e-k & $b-e$ \\
\hline \multirow{2}{*}{ G 04} & 67.5 & 89.5 & 94.5 & 27.0 & 5.00 & 24.40 & 31.35 & 48.6 & 44.2 & 805.0 & 14.31 & 285.0 & 49.1 & 40.25 & 385.0 \\
\hline & $\mathrm{a}$ & $b-d$ & $\mathrm{c}$ & $f-i$ & b-d & $b-f$ & $e-i$ & a-e & $\mathrm{b}-\mathrm{g}$ & $\mathrm{g}$ & b-g & $\mathrm{e}$ & $\mathrm{bc}$ & $\mathrm{c}-\mathrm{h}$ & b-e \\
\hline \multirow{2}{*}{ G 05} & 62.0 & 87.0 & 92.0 & 30.0 & 5.75 & 23.90 & 31.65 & 48.5 & 46.1 & 1037.5 & 15.00 & 395.0 & 38.3 & 41.95 & 450.0 \\
\hline & $\mathrm{h}$ & $\mathrm{f}-\mathrm{h}$ & ef & $a-c$ & $a b$ & $c-f$ & $c-f$ & a-e & a-e & $\mathrm{ab}$ & $a-e$ & $\mathrm{ab}$ & $\mathrm{c}$ & b-e & $\mathrm{ab}$ \\
\hline \multirow{2}{*}{ G 06} & 65.5 & 90.0 & 94.5 & 29.0 & 6.00 & 24.48 & 32.35 & 49.7 & 46.6 & 1022.5 & 14.51 & 337.5 & 46.7 & 39.18 & 420.0 \\
\hline & b-e & $a-c$ & c & b-e & a & b-e & a-e & $a-d$ & a-e & $a-c$ & $b-f$ & b-e & $\mathrm{bc}$ & $d-i$ & $a-d$ \\
\hline \multirow{2}{*}{ G 07} & 64.5 & 87.5 & 93.0 & 28.5 & 4.25 & 23.68 & 30.45 & 51.0 & 47.6 & 930.0 & 11.31 & 315.0 & 51.0 & 42.80 & 322.5 \\
\hline & d-g & e-h & $c-f$ & $c-f$ & d & $\mathrm{d}-\mathrm{f}$ & hi & $\mathrm{a}$ & $a-d$ & b-g & $\mathrm{g}$ & $b-e$ & $\mathrm{bc}$ & b-d & $\mathrm{e}$ \\
\hline \multirow[t]{2}{*}{ G 08} & 65.0 & 88.5 & 92.5 & 27.5 & 5.00 & 23.28 & 32.70 & 48.1 & 41.1 & 877.5 & 14.37 & 327.5 & 55.7 & 34.58 & 395.0 \\
\hline & $c-f$ & $c-f$ & $d-f$ & e-h & b-d & ef & $a-c$ & a-e & e-h & $\mathrm{c}-\mathrm{g}$ & b-g & b-e & $\mathrm{b}$ & $\mathrm{j}-1$ & b-e \\
\hline \multirow{2}{*}{ G 09} & 65.0 & 88.0 & 92.5 & 27.5 & 5.75 & 23.05 & 30.35 & 46.5 & 40.9 & 937.5 & 13.25 & 365.0 & 45.0 & 39.33 & 365.0 \\
\hline & $c-f$ & d-g & d-f & e-h & $a b$ & ef & i & b-e & e-h & b-g & c-g & a-e & $\mathrm{bc}$ & $\mathrm{d}-\mathrm{i}$ & b-e \\
\hline \multirow{2}{*}{ G 10} & 65.5 & 90.5 & 94.0 & 28.5 & 4.75 & 24.05 & 31.95 & 48.6 & 44.8 & 867.5 & 13.24 & 375.0 & 42.6 & 39.75 & 377.5 \\
\hline & b-e & $a b$ & $\mathrm{~cd}$ & $c-f$ & $\mathrm{~cd}$ & $c-f$ & b-f & a-e & $a-f$ & d-g & $\mathrm{c}-\mathrm{g}$ & $a-d$ & $\mathrm{bc}$ & $c-i$ & b-e \\
\hline \multirow{2}{*}{ G 11} & 63.0 & 86.5 & 92.5 & 29.5 & 5.00 & 24.33 & 33.10 & 51.3 & 50.4 & 795.0 & 11.52 & 297.5 & 45.7 & 41.25 & 340.0 \\
\hline & gh & gh & $d-f$ & $a-d$ & b-d & $b-f$ & a & a & $a b$ & $\mathrm{~g}$ & $\mathrm{fg}$ & $\mathrm{de}$ & $\mathrm{bc}$ & $c-f$ & $c-e$ \\
\hline \multirow{2}{*}{ G 12} & 67.0 & 88.0 & 93.0 & 26.0 & 6.00 & 24.43 & 33.30 & 47.2 & 43.0 & 982.5 & 16.73 & 315.0 & 55.0 & 33.90 & 435.0 \\
\hline & $a b$ & d-g & $c-f$ & $h-j$ & $\mathrm{a}$ & $b-f$ & a & a-e & c-g & $b-f$ & $a b$ & b-e & $\mathrm{b}$ & $\mathrm{kl}$ & $a b$ \\
\hline \multirow{2}{*}{ G 13} & 66.0 & 89.0 & 93.0 & 27.0 & 5.25 & 23.58 & 31.60 & 45.6 & 45.3 & 922.5 & 14.72 & 352.5 & 46.4 & 47.70 & 397.5 \\
\hline & $a-d$ & b-e & $c-f$ & $f-i$ & $a-c$ & $d-f$ & d-g & de & $a-f$ & b-g & $b-e$ & a-e & bc & $\mathrm{a}$ & b-e \\
\hline \multirow{2}{*}{ G 14} & 67.5 & 90.5 & 93.0 & 25.5 & 6.00 & 23.25 & 31.98 & 50.3 & 45.6 & 1005.0 & 16.11 & 327.5 & 55.4 & 38.93 & 410.0 \\
\hline & $\mathrm{a}$ & $a b$ & $c-f$ & ij & a & ef & b-f & $a-c$ & $a-f$ & a-e & $a-c$ & $b-e$ & $\mathrm{~b}$ & $d-j$ & $a-d$ \\
\hline G 15 & 64.5 & 88.5 & 92.0 & 27.5 & 5.25 & 24.03 & 31.25 & 47.2 & 45.3 & 840.0 & 12.29 & 305.0 & 53.0 & 36.63 & 337.5 \\
\hline U 15 & d-g & $c-f$ & ef & $e-h$ & $a-c$ & $c-f$ & $f-i$ & a-e & $a-f$ & fg & e-g & $c-e$ & $\mathrm{~b}$ & $\mathrm{~g}-1$ & de \\
\hline G 16 & 63.5 & 88.5 & 94.5 & 31.0 & 5.75 & 24.98 & 31.40 & 50.5 & 50.6 & 902.5 & 12.66 & 285.0 & 72.3 & 33.13 & 392.5 \\
\hline 410 & $\mathrm{f}-\mathrm{h}$ & $c-f$ & $\mathrm{bc}$ & $\mathrm{a}$ & $a b$ & $a-d$ & e-h & $a b$ & $\mathrm{a}$ & b-g & e-g & $\mathrm{e}$ & $\mathrm{a}$ & 1 & $b-e$ \\
\hline G 17 & 66.0 & 90.0 & 96.0 & 30.0 & 5.25 & 23.58 & 32.88 & 49.1 & 44.5 & 1017.5 & 15.00 & 377.5 & 46.1 & 36.55 & 450.0 \\
\hline U 17 & $a-d$ & $a-c$ & $\mathrm{ab}$ & $a-c$ & $a-c$ & $d-f$ & $a b$ & a-e & $a-g$ & $a-d$ & a-e & $a-d$ & $\mathrm{bc}$ & $\mathrm{g}-1$ & $\mathrm{ab}$ \\
\hline G 18 & 66.5 & 90.5 & 97.0 & 30.5 & 6.00 & 24.25 & 31.53 & 47.2 & 45.5 & 920.0 & 12.80 & 332.5 & 47.0 & 41.00 & 390.0 \\
\hline 410 & $a-c$ & $a b$ & $\mathrm{a}$ & $a b$ & $\mathrm{a}$ & $c-f$ & d-g & a-e & $a-f$ & b-g & d-g & b-e & $\mathrm{bc}$ & $c-g$ & b-e \\
\hline G 19 & 66.0 & 89.5 & 94.5 & 28.5 & 5.75 & 22.98 & 32.35 & 50.9 & 48.4 & 905.0 & 14.02 & 360.0 & 51.2 & 35.45 & 400.0 \\
\hline 419 & a-d & $b-d$ & $\mathrm{bc}$ & $c-f$ & $a b$ & $\mathrm{f}$ & a-e & $\mathrm{a}$ & $a-c$ & b-g & b-g & $a-e$ & $\mathrm{bc}$ & i-1 & b-e \\
\hline G 20 & 64.0 & 86.0 & 92.0 & 28.0 & 5.75 & 23.90 & 32.30 & 49.6 & 45.0 & 870.0 & 13.04 & 322.5 & 43.2 & 33.20 & 365.0 \\
\hline & e-g & $\mathrm{h}$ & ef & d-g & $a b$ & $c-f$ & $a-f$ & $a-d$ & $a-f$ & $c-g$ & $\mathrm{c}-\mathrm{g}$ & b-e & $\mathrm{bc}$ & 1 & b-e \\
\hline G 21 & 67.5 & 90.0 & 93.5 & 26.0 & 5.35 & 23.73 & 32.90 & 49.3 & 47.8 & 862.5 & 13.17 & 307.5 & 46.1 & 33.93 & 342.5 \\
\hline$G 21$ & $\mathrm{a}$ & $a-c$ & c-e & $h-j$ & $a-c$ & $d-f$ & $a b$ & a-e & $a-d$ & e-g & $c-g$ & $c-e$ & $\mathrm{bc}$ & $\mathrm{kl}$ & $\mathrm{c}-\mathrm{e}$ \\
\hline G 22 & 64.0 & 86.5 & 91.5 & 27.5 & 5.35 & 23.03 & 30.58 & 50.2 & 46.5 & 1150.0 & 17.90 & 385.0 & 52.3 & 37.38 & 492.5 \\
\hline $\mathrm{G} 22$ & e-g & $\mathrm{gh}$ & f & $e-h$ & $a-c$ & ef & $g-i$ & $a-c$ & a-e & $\mathrm{a}$ & $\mathrm{a}$ & $a-c$ & $\mathrm{bc}$ & $\mathrm{f}-1$ & $\mathrm{a}$ \\
\hline G 23 & 64.0 & 86.0 & 90.0 & 26.0 & 5.00 & 24.83 & 33.35 & 46.2 & 38.5 & 917.5 & 15.96 & 432.5 & 56.1 & 36.35 & 415.0 \\
\hline$U 23$ & e-g & $\mathrm{h}$ & $\mathrm{g}$ & $h-j$ & b-d & $b-d$ & a & $c-e$ & gh & b-g & $a-d$ & $\mathrm{a}$ & $\mathrm{b}$ & h-1 & $a-d$ \\
\hline G 24 & 66.0 & 90.0 & 93.5 & 27.5 & 5.90 & 24.35 & 32.53 & 49.6 & 46.7 & 987.5 & 15.44 & 397.5 & 42.8 & 39.35 & 425.0 \\
\hline & $a-d$ & $a-c$ & $c-e$ & $\mathrm{e}-\mathrm{h}$ & $\mathrm{a}$ & $b-f$ & $a-d$ & $a-d$ & $a-e$ & $b-f$ & a-e & $a b$ & $\mathrm{bc}$ & $\mathrm{d}-\mathrm{i}$ & $a-c$ \\
\hline G 25 & 67.0 & 87.5 & 92.0 & 25.0 & 5.75 & 25.28 & 33.05 & 45.7 & 39.6 & 840.0 & 14.70 & 302.5 & 54.7 & 33.30 & 367.5 \\
\hline$G 25$ & $a b$ & $\mathrm{e}-\mathrm{h}$ & ef & $\mathrm{j}$ & $a b$ & $a-c$ & $\mathrm{a}$ & de & $\mathrm{f}-\mathrm{h}$ & $\mathrm{fg}$ & b-e & c-e & $\mathrm{b}$ & 1 & b-e \\
\hline Mean & 65.4 & 88.6 & 93.3 & 27.9 & 5.43 & 24.19 & 32.05 & 48.5 & 44.6 & 933.2 & 14.32 & 340.6 & 49.6 & 38.54 & 398.4 \\
\hline $\begin{array}{l}\% \text { Red } \\
\text { uction }\end{array}$ & 6.3 & 12.2 & 13.3 & 26.2 & & & & & 6.4 & 19.6 & & 9.2 & 10.1 & 8.68 & 22.5 \\
\hline$\%$ Incr & ase & & & & 17.79 & 13.41 & 27.36 & 3.91 & & & 5.04 & & & & \\
\hline
\end{tabular}

Means of the same column followed by the same letter do not differ significantly at $5 \%$ level of probability $\mathrm{FLS}=$ Flag leaf senescence (day); PM=Physiological maturity (day); GFD=Grain filling duration (day); $\mathrm{GC}_{35}=$ Ground coverage at 35 days; $\mathrm{CT}_{\mathrm{vg}}=$ Canopy temperature at vegetative stage $\left({ }^{\circ} \mathrm{C}\right) ; \mathrm{CTgf}=$ Canopy temperature at grain filling stage $\left({ }^{\circ} \mathrm{C}\right) ; \mathrm{CHL}_{\mathrm{a}}=$ Chlorophyll content at anthesis (spad unit); $\mathrm{CHL}_{21}=$ Chlorophyll content at 21 days after anthesis ( ppad unit); Bio $\mathrm{m}^{-2}=$ Biomass $\mathrm{m}^{-2}$; GFR $=$ Grain filling rate $\left(\mathrm{g} \mathrm{m}^{-2} \mathrm{~d}^{-1}\right)$ 
ence of genes for heat tolerance. Rasal et al. (2006) and Amandeep et al. (2007) found $17.45 \%$ and $28.9 \%$ yield reduction, respectively in response to heat stress, though Modarresi et al. (2010) showed the highest $(46.63 \%)$ yield reduction. In spite of this, it was noticed that the genotypes G-1, G-2, G-5, G-6, G-12, G-14, G-17, G-22, G-23 and G-24 had yielded higher grain even under heat stressed condition.

\section{Identification of heat tolerant genotypes through heat stress susceptibility indices}

Paired t-test was employed to detect the differences between the effect of heat stress on these genotypes (Table 3). Significant differences between optimum sowing and late sowing environments could be found for all the characters except grain filling rate. In the present study, grain filling rate was excluded for preparing susceptibility indices as it showed insignificant response in paired t-test. Heat stress susceptibility indices " $\mathrm{S}$ " were measured for the characters to identify heat susceptible or tolerant genotypes. It adjusts for variations in grain yield, yield contributing, phenological and physiological traits due to differences in environmental stress intensity. The genotypes showed wide range of variations for "S" values. These values were used for identifying heat tolerant genotypes. Low stress susceptibility $(\mathrm{S}<1)$ is synonymous with high stress tolerance (Fischer and Maurer 1978). Based upon the value and direction of desirability, different genotypes were ranked as highly heat stress tolerant $(\mathrm{S}<0.50)$, moderately heat stress tolerant $(\mathrm{S}>0.50<1.00)$ and heat susceptible $(\mathrm{S}>1.00)$ (Khanna-Chopra and Viswanathan 1999 and Singh et al. 2011). Thus, in order to determine relative tolerance, the heat susceptibility indices were estimated for various characters. Several authors (Khanna-Chopra and Viswanathan 1999; Singh et al. 2011 and Sharma et al. 2013) evaluated heat susceptibility indices of yield and its different components of wheat genotypes for heat stress tolerance and grouped them into highly tolerant, tolerant and susceptible genotypes as suggested by low and high $\mathrm{S}$ values. The present findings revealed that heat susceptibility indices could be taken as important criteria for breeding wheat genotypes suitable for late sowing conditions. Considering heat stress intensity, it was revealed that days to anthesis, chlorophyll content of flag leaf, number of spikes $\mathrm{m}^{-2}$ and 1000-grain weight were less affected by late sowing condition, while ground cover, grain filling duration, canopy temperature, biomass and grain yield highly suffered under late sowing environment. This indicates that wheat grain yield depends on its filling duration and canopy structure under late sowing condition which partially supported the findings of Singh et al. (2011).

Estimation of heat stress susceptibility indices and raking of genotypes (Table 4) showed that every genotype possess different degree of tolerance to heat stress. According to heat stress susceptibility indices estimated for anthesis period, 13 among 25 genotypes (G-5, G -20, G-11, G-22, G-3, G-18, G-13, G-2, G-19, G-24, G-6, G-8 and G-7) were identified as tolerant and 4 of them were found highly tolerant $(S<0.5)$. The rest of the genotypes including check were found to be heat sensitive. Ten genotypes including check G-5, G-6, G1, G-20, G-3, G-17, G-18, G-23, G-11 and G7 were found to be tolerant based on "S" values for physiological maturity. Based on "S" values for grain filling duration, 11 genotypes (G-21, G-1, G-6, G-4, G-23, G-16, G-17, G25, G-18, G-5 and G-7) seemed to be moderately tolerant $(S<1>0.5)$. Moreover 11 genotypes (G-6, G-16, G-15, G-2, G-10, G-7, G-3, G-4, G-1, G-19 and G-20) showed "S" values less than unity for the estimation of ground coverage while first 6 of them were highly tolerant $(\mathrm{S}<0.5)$.

The estimates on "S" values for canopy temperature at vegetative stage revealed that among 25 genotypes, 13 including check (G15, G-12, G-17, G-14, G-13, G-21, G-25, G- 
11, G-16, G-24, G-19, G-18 and G-1) showed tolerance to late sowing. The genotypes G-15 and G-12 were found to be highly tolerant $(\mathrm{S}<0.5)$ for this trait. However, fourteen genotypes (G-3, G-15, G-2, G-14, G-16, G-7, G-4, G-1, G-5, G-22, G-13, G-25, G-9 and G-19) showed moderate tolerance $(\mathrm{S}<1>0.5)$ based on "S" values for canopy temperature at grain filling. Estimated " $S$ " values for chlorophyll content measured at anthesis showed that fourteen genotypes (G-16, G-14, G-12, G-22, G-4, G-9, G-2, G-3, G-6, G-18, G-21, G-8, G10 and G-11) were found to be tolerant of which first 8 genotypes were considered as highly tolerant $(\mathrm{S}<0.5)$.

"S" values for spikes number $\mathrm{m}^{-2}$ showed that 14 genotypes including check G-7, G-23, G9, G-1, G-14, G-3, G-19, G-13, G-22, G-11, G-5, G-21, G-25 and G-18 were tolerant of which first 10 genotypes were considered as highly tolerant $(\mathrm{S}<0.5)$. The estimates of " $\mathrm{S}$ " values for the number of grains spike ${ }^{-1}$ revealed that 16 genotypes including check G1, G-15, G-11, G-7, G-21, G-16, G-13, G-3,
G-17, G-20, G-8, G-23, G-14, G-19, G-12 and G-18 displayed tolerance to heat stress of which 7 genotypes were considered as highly tolerant $(\mathrm{S}<0.5)$. Besides, 13 genotypes $\mathrm{G}-10$, G-18, G-14, G-4, G-19, G-1, G-12, G-9, G-7, G-23, G-25, G-15 and G-6 were identified as tolerant based on "S" values when estimated for thousand grain weight. Among them, 6 genotypes including check were considered as highly tolerant $(\mathrm{S}<0.5)$. Moreover the " $\mathrm{S}$ " values of 14 genotypes including check G-14, G12, G-13, G-25, G-2, G-23, G-18, G-17, G-24, G-6, G-19, G-1, G-9 and G-22 were observed as tolerant when estimated for grain yield. The genotypes G-14 and G-12 were considered as highly tolerant $(\mathrm{S}<0.5)$ and rest of the genotypes showed moderate tolerance to grain yield. Several genotypes showed less heat sensitivity to both grain yield and 1000-grain weight. Among 25 genotypes, the genotypes G-14, G-12, G-25 and G-13 showed both tolerances to heat stress for these two traits with moderate yield.

Table 3 Paired t-test for each character using the accession means of optimum sowing and late sowing conditions

\begin{tabular}{|c|c|c|c|c|c|}
\hline $\begin{array}{l}\text { Sl. } \\
\text { no. }\end{array}$ & Character & $\begin{array}{l}\text { Mean difference } \\
\bar{d}\end{array}$ & $\begin{array}{l}\text { Standard } \\
\text { deviation } \\
\left(\mathrm{s}_{\mathrm{d}}\right)\end{array}$ & t-value & $\begin{array}{l}\text { Significance } \\
\text { level }\end{array}$ \\
\hline$\overline{1}$ & Days to Anthesis & 4.44 & 2.14 & 10.33 & $* *$ \\
\hline 2 & Flag leaf senescence (day) & 12.40 & 1.19 & 51.71 & $* *$ \\
\hline 3 & Physiological maturity (day) & 14.36 & 1.63 & 43.87 & $* *$ \\
\hline 4 & Grain filling duration (day) & 9.92 & 1.94 & 25.56 & $* *$ \\
\hline 5 & Ground coverage at 35 days (scale) & 0.82 & 0.44 & 9.28 & $* *$ \\
\hline 6 & Canopy temperature at veg. stage $\left({ }^{0} \mathrm{C}\right)$ & 2.86 & 1.04 & 13.66 & $* *$ \\
\hline 7 & Canopy temperature at grain filling $\left({ }^{0} \mathrm{C}\right)$ & 6.88 & 1.02 & 33.75 & $* *$ \\
\hline 8 & Chlorophyll content at anthesis (spad) & 1.82 & 1.48 & 6.14 & $* *$ \\
\hline 9 & Chlorophyll content at 21 DAA (spad) & 3.06 & 2.43 & 6.28 & $* *$ \\
\hline 10 & Biomass $\mathrm{m}^{-2}(\mathrm{~g})$ & 228.20 & 118.04 & 9.66 & $* *$ \\
\hline 11 & Grain filling rate $\left(\mathrm{g} \mathrm{m}^{-2} \mathrm{~d}^{-1}\right)$ & 0.68 & 1.86 & 1.84 & ns \\
\hline 12 & Spikes number $\mathrm{m}^{-2}$ & 34.90 & 32.78 & 5.32 & $* *$ \\
\hline 13 & Grains number spike $e^{-1}$ & 5.62 & 4.38 & 6.41 & $* *$ \\
\hline 14 & 1000-grains weight $(\mathrm{g})$ & 3.66 & 3.02 & 6.05 & $* *$ \\
\hline 15 & Grain yield $\mathrm{m}^{-2}(\mathrm{~g})$ & 116.00 & 56.08 & 10.34 & $* *$ \\
\hline
\end{tabular}

** indicate significant at $1 \%$ level of probability; ns: non significant 
Table 4 Heat stress susceptibility indices " $S$ " based on phenological, physiological and primary yield contribut-

\begin{tabular}{|c|c|c|c|c|c|c|c|c|c|c|c|c|c|c|}
\hline \multirow[b]{2}{*}{ Genotype } & \multicolumn{2}{|c|}{ Anthesis } & \multicolumn{2}{|l|}{ FLS } & \multicolumn{2}{|l|}{ PM } & \multicolumn{2}{|c|}{ GFD } & \multicolumn{2}{|c|}{$\mathrm{GC}_{35}$} & \multicolumn{2}{|l|}{ CTvg } & \multicolumn{2}{|l|}{ CTgf } \\
\hline & $\overline{\mathrm{S}_{\mathrm{A}}}$ & Rank & $\mathrm{S}_{\mathrm{FLS}}$ & Rank & $\mathrm{S}_{\mathrm{PM}}$ & Rank & $\overline{\mathrm{S}_{\mathrm{GFD}}}$ & $\begin{array}{l}\text { Ran } \\
\mathrm{k}\end{array}$ & $\mathrm{S}_{\mathrm{GC}}$ & Rank & $\mathrm{S}_{\mathrm{CTVG}}$ & Rank & $\mathrm{S}_{\mathrm{CTGF}}$ & Rank \\
\hline G 01(ck.) & 1.12 & 17 & 0.77 & 1 & 0.86 & 3 & 0.75 & 2 & 0.85 & 9 & 0.99 & 13 & 0.96 & 8 \\
\hline G 02 & 0.90 & 8 & 0.97 & 7 & 1.04 & 17 & 1.10 & 19 & 0.31 & 4 & 1.60 & 24 & 0.78 & 3 \\
\hline G 03 & 0.79 & 5 & 0.86 & 3 & 0.92 & 5 & 1.01 & 12 & 0.56 & 7 & 1.43 & 21 & 0.76 & 1 \\
\hline G 04 & 1.57 & 23 & 1.03 & 16 & 1.00 & 11 & 0.78 & 4 & 0.63 & 8 & 1.54 & 23 & 0.88 & 7 \\
\hline G 05 & 0.00 & 1 & 0.95 & 5 & 0.77 & 1 & 0.99 & 10 & 1.19 & 15 & 1.75 & 25 & 0.97 & 9 \\
\hline G 06 & 0.91 & 11 & 0.82 & 2 & 0.81 & 2 & 0.78 & 3 & 0.25 & 1 & 1.03 & 15 & 1.07 & 20 \\
\hline G 07 & 0.92 & 13 & 0.95 & 4 & 0.98 & 10 & 0.99 & 11 & 0.35 & 6 & 1.45 & 22 & 0.88 & 6 \\
\hline G 08 & 0.91 & 12 & 0.97 & 8 & 1.02 & 14 & 1.05 & 16 & 1.00 & 12 & 1.13 & 16 & 1.44 & 25 \\
\hline G 09 & 1.12 & 18 & 1.08 & 22 & 1.08 & 21 & 1.05 & 17 & 1.19 & 16 & 1.26 & 17 & 0.98 & 13 \\
\hline G 10 & 1.01 & 15 & 0.99 & 9 & 1.03 & 16 & 1.03 & 13 & 0.31 & 5 & 1.01 & 14 & 1.02 & 16 \\
\hline G 11 & 0.25 & 3 & 0.99 & 10 & 0.96 & 9 & 1.13 & 20 & 1.41 & 19 & 0.77 & 8 & 1.28 & 23 \\
\hline G 12 & 1.09 & 16 & 1.05 & 18 & 1.07 & 19 & 1.10 & 18 & 1.49 & 21 & 0.40 & 2 & 1.11 & 21 \\
\hline G 13 & 0.79 & 7 & 1.04 & 17 & 1.07 & 20 & 1.17 & 23 & 1.33 & 17 & 0.71 & 5 & 0.97 & 11 \\
\hline G 14 & 1.48 & 22 & 1.06 & 20 & 1.13 & 24 & 1.03 & 15 & 1.13 & 14 & 0.57 & 4 & 0.79 & 4 \\
\hline G 15 & 1.24 & 19 & 1.15 & 25 & 1.23 & 25 & 1.19 & 24 & 0.28 & 3 & 0.13 & 1 & 0.76 & 2 \\
\hline G 16 & 1.46 & 21 & 1.11 & 24 & 1.03 & 15 & 0.82 & 6 & 0.26 & 2 & 0.79 & 9 & 0.87 & 5 \\
\hline G 17 & 1.00 & 14 & 0.99 & 12 & 0.92 & 6 & 0.88 & 7 & 1.33 & 18 & 0.55 & 3 & 1.05 & 18 \\
\hline G 18 & 0.79 & 6 & 1.02 & 15 & 0.95 & 7 & 0.98 & 9 & 1.49 & 22 & 0.95 & 12 & 1.00 & 15 \\
\hline G 19 & 0.90 & 9 & 0.96 & 6 & 1.00 & 12 & 1.03 & 14 & 0.85 & 10 & 0.94 & 11 & 0.99 & 14 \\
\hline G 20 & 0.12 & 2 & 1.07 & 21 & 0.90 & 4 & 1.14 & 21 & 0.85 & 11 & 1.32 & 18 & 1.17 & 22 \\
\hline G 21 & 1.76 & 25 & 1.10 & 23 & 1.01 & 13 & 0.71 & 1 & 1.07 & 13 & 0.72 & 6 & 1.06 & 19 \\
\hline G 22 & 0.48 & 4 & 0.99 & 11 & 1.06 & 18 & 1.22 & 25 & 1.91 & 23 & 1.39 & 20 & 0.97 & 10 \\
\hline G 23 & 1.35 & 20 & 1.00 & 14 & 0.95 & 8 & 0.81 & 5 & 1.41 & 20 & 1.38 & 19 & 1.31 & 24 \\
\hline G 24 & 0.90 & 10 & 0.99 & 13 & 1.10 & 22 & 1.16 & 22 & 2.68 & 25 & 0.86 & 10 & 1.04 & 17 \\
\hline G 25 & 1.68 & 24 & 1.05 & 19 & 1.11 & 23 & 0.92 & 8 & 1.99 & 24 & 0.75 & 7 & 0.97 & 12 \\
\hline "S" Range & \multicolumn{2}{|c|}{$0.00-1.76$} & \multicolumn{2}{|c|}{$0.77-1.15$} & \multicolumn{2}{|c|}{$0.77-1.23$} & \multicolumn{2}{|c|}{$0.71-1.22$} & \multicolumn{2}{|c|}{$0.25-2.68$} & \multicolumn{2}{|c|}{$0.13-1.75$} & \multicolumn{2}{|l|}{$0.76-1.44$} \\
\hline Stress Intensity & \multicolumn{2}{|c|}{0.063} & \multicolumn{2}{|c|}{0.122} & \multicolumn{2}{|c|}{0.133} & \multicolumn{2}{|c|}{0.262} & \multicolumn{2}{|c|}{-0.177} & \multicolumn{2}{|c|}{-0.134} & \multicolumn{2}{|l|}{-0.273} \\
\hline
\end{tabular}

$\mathrm{A}=$ Days to anthesis; FLS= Flag leaf senescence (day); $\mathrm{PM}=$ Physiological maturity (day); $\mathrm{GFD}=$ Grain filling duration (day); $\mathrm{GC}_{35}=$ Ground coverage at 35 days; $\mathrm{CT}_{\mathrm{vg}}=$ Canopy temperature at vegetative stage $\left({ }^{\circ} \mathrm{C}\right)$; $\mathrm{CTgf}=$ Canopy temperature at grain filling stage $\left({ }^{\circ} \mathrm{C}\right)$

Table 4 Cont'd

\begin{tabular}{|c|c|c|c|c|c|c|c|c|c|c|c|c|c|c|}
\hline \multirow[b]{2}{*}{ Genotype } & \multicolumn{2}{|c|}{ CHLa } & \multicolumn{2}{|l|}{$\mathrm{CHL}_{21}$} & \multicolumn{2}{|c|}{ Bio $\mathrm{m}^{-2}$} & \multicolumn{2}{|c|}{ Spikes $\mathrm{m}^{-2}$} & \multicolumn{2}{|c|}{ Grains sp ${ }^{-1}$} & \multicolumn{2}{|c|}{$\begin{array}{l}\text { 1000-grain } \\
\text { wt. }\end{array}$} & \multicolumn{2}{|c|}{$\begin{array}{l}\text { Grain Yield } \\
\mathrm{m}^{-2}\end{array}$} \\
\hline & $\begin{array}{l}\mathrm{S}_{\mathrm{CH}} \\
\mathrm{A}\end{array}$ & Rank & $\mathrm{S}_{\mathrm{CH} 21}$ & $\begin{array}{l}\text { Ran } \\
\mathrm{k}\end{array}$ & $\overline{\mathrm{S}_{\mathrm{B}}}$ & $\begin{array}{l}\text { Ran } \\
\mathrm{k}\end{array}$ & $\overline{\mathrm{S}_{\mathrm{SM}}}$ & Rank & $\mathrm{S}_{\mathrm{GS}}$ & Rank & $\overline{\mathrm{S}_{\mathrm{TGW}}}$ & $\begin{array}{l}\text { Ran } \\
\mathrm{k}\end{array}$ & $\overline{\mathrm{S}_{\mathrm{GY}}}$ & Rank \\
\hline G 01(ck.) & 2.02 & 22 & 1.99 & 21 & 1.05 & 14 & 0.16 & 4 & 0.10 & 1 & 0.31 & 6 & 0.92 & 12 \\
\hline G 02 & 0.41 & 7 & 2.28 & 23 & 0.63 & 7 & 1.64 & 19 & 1.40 & 18 & 1.63 & 23 & 0.64 & 5 \\
\hline G 03 & 0.49 & 8 & 3.01 & 25 & 1.27 & 21 & 0.17 & 6 & 0.54 & 8 & 1.15 & 17 & 1.04 & 15 \\
\hline G 04 & 0.19 & 5 & 1.13 & 17 & 1.61 & 23 & 2.26 & 23 & 2.26 & 24 & 0.18 & 4 & 1.50 & 23 \\
\hline G 05 & 2.08 & 23 & 0.59 & 10 & 1.00 & 12 & 0.52 & 11 & 2.53 & 25 & 1.09 & 15 & 1.07 & 16 \\
\hline G 06 & 0.50 & 9 & 1.06 & 15 & 0.47 & 3 & 2.34 & 25 & 1.94 & 22 & 0.93 & 13 & 0.85 & 10 \\
\hline G 07 & 1.05 & 16 & 0.24 & 5 & 1.11 & 17 & 0.00 & 1 & 0.16 & 4 & 0.62 & 9 & 1.70 & 24 \\
\hline G 08 & 0.68 & 12 & 1.22 & 18 & 1.89 & 25 & 2.29 & 24 & 0.68 & 11 & 1.89 & 24 & 1.39 & 21 \\
\hline G 09 & 0.28 & 6 & 2.39 & 24 & 0.80 & 9 & 0.15 & 3 & 2.13 & 23 & 0.62 & 8 & 0.95 & 13 \\
\hline G 10 & 0.76 & 13 & 1.32 & 19 & 1.26 & 20 & 2.24 & 22 & 1.67 & 20 & 0.04 & 1 & 1.10 & 17 \\
\hline G 11 & 0.90 & 14 & 0.03 & 1 & 1.20 & 18 & 0.44 & 10 & 0.16 & 3 & 1.30 & 20 & 1.17 & 19 \\
\hline G 12 & 0.14 & 3 & 0.25 & 6 & 0.42 & 2 & 1.98 & 20 & 0.89 & 15 & 0.57 & 7 & 0.24 & 2 \\
\hline
\end{tabular}


Tropical Agricultural Research \& Extension 17(1): 2014

\begin{tabular}{|c|c|c|c|c|c|c|c|c|c|c|c|c|c|c|}
\hline G 13 & 3.30 & 25 & 0.69 & 11 & 0.48 & 4 & 0.23 & 8 & 0.44 & 7 & 1.05 & 14 & 0.50 & 3 \\
\hline G 14 & 0.03 & 2 & 0.95 & 14 & 0.05 & 1 & 0.16 & 5 & 0.74 & 13 & 0.15 & 3 & 0.23 & 1 \\
\hline G 15 & 1.02 & 15 & 1.12 & 16 & 1.31 & 22 & 1.05 & 16 & 0.12 & 2 & 0.93 & 12 & 1.46 & 22 \\
\hline G 16 & 0.03 & 1 & 0.21 & 4 & 1.23 & 19 & 1.04 & 15 & 0.28 & 6 & 1.24 & 19 & 1.17 & 18 \\
\hline G 17 & 1.29 & 18 & 0.80 & 12 & 0.58 & 6 & 1.60 & 18 & 0.59 & 9 & 1.54 & 22 & 0.74 & 8 \\
\hline G 18 & 0.56 & 10 & 0.55 & 9 & 0.81 & 10 & 0.76 & 14 & 0.94 & 16 & 0.06 & 2 & 0.74 & 7 \\
\hline G 19 & 2.76 & 24 & 0.36 & 7 & 1.06 & 15 & 0.22 & 7 & 0.75 & 14 & 0.19 & 5 & 0.87 & 11 \\
\hline G 20 & 1.98 & 21 & 1.49 & 20 & 1.80 & 24 & 2.05 & 21 & 0.59 & 10 & 1.37 & 21 & 1.84 & 25 \\
\hline G 21 & 0.64 & 11 & 0.11 & 2 & 1.01 & 13 & 0.59 & 12 & 0.25 & 5 & 3.44 & 25 & 1.20 & 20 \\
\hline G 22 & 0.15 & 4 & 0.83 & 13 & 1.06 & 16 & 0.34 & 9 & 1.92 & 21 & 1.23 & 18 & 0.98 & 14 \\
\hline G 23 & 1.68 & 20 & 2.05 & 22 & 0.77 & 8 & 0.00 & 2 & 0.74 & 12 & 0.77 & 10 & 0.72 & 6 \\
\hline G 24 & 1.68 & 19 & 0.12 & 3 & 0.92 & 11 & 1.05 & 17 & 1.40 & 19 & 1.13 & 16 & 0.83 & 9 \\
\hline G 25 & 1.17 & 17 & 0.38 & 8 & 0.53 & 5 & 0.59 & 13 & 1.30 & 17 & 0.77 & 11 & 0.51 & 4 \\
\hline "S" Range & \multicolumn{2}{|c|}{$0.03-3.30$} & \multicolumn{2}{|c|}{$0.03-3.01$} & \multicolumn{2}{|c|}{$0.05-1.89$} & \multicolumn{2}{|c|}{$0.00-2.34$} & \multicolumn{2}{|c|}{$0.10-2.53$} & \multicolumn{2}{|c|}{$0.04-3.44$} & \multicolumn{2}{|c|}{$0.23-1.84$} \\
\hline \multicolumn{3}{|c|}{ Stress Intensity -0.039} & \multicolumn{2}{|c|}{0.064} & \multicolumn{2}{|c|}{0.196} & \multicolumn{2}{|c|}{0.092} & \multicolumn{2}{|c|}{0.101} & \multicolumn{2}{|c|}{0.086} & \multicolumn{2}{|c|}{0.225} \\
\hline
\end{tabular}

$\mathrm{CHL}_{\mathrm{a}}=$ Chlorophyll content at anthesis (Spad unit); $\mathrm{CHL}_{21}=$ Chlorophyll content at 21 days after anthesis (Spad unit); Bio $\mathrm{m}^{-2}=$ Biomass $\mathrm{m}^{-2}$

Based on overall results, G-18, G-19, G-14, G -12 , G-13, G-6, G-25, G-23, G-9 and check (G-1) could be categorized as heat tolerance genotypes. Among them, G-18, G-19, G-14, G-12 and G-13 were performed better than the check (G-1). High stability in grain yield under stress condition was associated with poor or moderate grain yield potential (Fischer and Maurer 1978; Bruckner and Frehberg 1987; Ehdaie et al. 1988; Bansal and Sinha 1991). In this study, the genotypes G14, G-12, G-25 and G-13 produced moderate grain yield and found to be highly stable genotypes under heat stress, thus could be recommended for both sowing environments while the genotypes G-2, G-17, G-22, and G24 might be recommended for optimum sowing condition with relatively high yield as they showed moderate tolerance to heat stress under late sowing condition. Screened genotypes for heat stress tolerance could potentially be used as a genetic stock for further improvements of genotypes for heat stress.

\section{REFERENCES}

Acevedo E, Nachit M and Ferrara M 1991 Effect of heat stress on wheat and possible selection tools for use in breeding for tolerance. In: Wheat for nontraditional warm areas, Saunders DA (ed.), Mexico, DF, CIMMYT.
Al-Karaki GN 2012 Phenological development-yield relationships in durum wheat cultivars under late-season hightemperature stress in a semiarid environment. International Scholarly Research Network, ISRN Agronomy, 2012 , ID 456856, page 7 . doi:10.5402/2012/456856.

Al-Khatib K and Paulsen GM 1999 High temperature effect on photosynthetic processes in temperate and tropical cereals. Crop Sci. 39: 119-125.

Almeselmani M, Abdullah F, Hareri F, Naaesan M, Ammar MA, Kanbar OZ 2011 Effect of drought on different physiological characters and yield component in different Syrian durum wheat varieties. J Agric. Sci. 3:127-33.

Amandeep K, Sohu VS and Mavi GS 2007 Genotypic variation for physiological traits associated with heat tolerance in bread wheat (Triticum aestivum L.). Crop-Improvement 34(2): 117-123.

Amani I, Fischer RA and Reynolds MP 1996 Canopy temperature depression association with yield of irrigated spring wheat cultivars in hot climate. J. Agron. Crop Sci. 176:119-129.

Ayeneh A, Ginkel M, Reynolds MP and Ammar K 2002 Comparison of leaf, spike, peduncle, and canopy temperature depression in wheat under heat stress. 
Field Crops Res. 79: 173-184.

Badaruddin M, Reynolds MP and. Ageeb OAA 1999 Wheat management in warm environments: effect of organic and inorganic fertilizers, irrigation frequency and mulching. Agron. J. 91:975 $-983$.

Bansal KC and Sinha SK 1991 Assessment of drought resistance in 20 accessions of Triticum aestivum and related species. I. Total dry matter and grain yiield stability. Euphytica 56: 7-14.

Barma NCD 2005 Genetic study of morphophysiological traits related to heat tolerance in spring wheat. Ph.D. Thesis, Department of Genetics and Plant Breeding, Bangladesh Agric. Univ. Mymensingh-2200, Bangladesh.

Barma NCD, Islam MA, Hakim MA and Sarker DKR 2011 Genetic variability and selection response to heat tolerance through membrane thermostability in spring wheat (Triticum aestivum L). Bangladesh J. P1. Breed. Genet. 23(2): 15-22.

Blum A, Sullivan CY and Naguyen HT 1997 The effect of plant size of wheat in responses to drought stress II. Water deficit, heat and ABA. Aust. J. Plant Physiol. 24: 43-48.

Bruckner PL and Frohberg RC 1987 Rate and duration of grain fill in spring wheat. Crop Sci. 27: 451-455.

CIMMYT 1995 CIMMYT/NARS Consultancy on ME: Bread Wheat Breeding. Wheat Special Report No. 38. Mexico, DF, CIMMYT.

DEFRA 2005 India-UK collaboration on impacts of climate change in India. http:// www.defra.gov.uk/environment/ climatechange/internat/devcountry/ india2.htm (accessed on 18 April 2006).

Dhyani K, Ansari MW, Rao YR, Verma RS, Shukla A and Tuteja N 2013 Comparative physiological response of wheat genotypes under terminal heat stress. Plant Signaling \& Behavior 8(6): 245264.
Ehdaie B, Waines JG and Hall AE 1988 Differential responses of landrace and improved spring wheat genotypes to stress environment. Crop Sci. 28: 838-842.

Fischer RA and Maurer O 1978 Crop temperature modification and yield potential in a dwarfing spring wheat. Crop Sci. 16: 855-859.

Gomez KA and Gomez A 1984 Statistical Procedures for Aagricultural Research (second edition), pp 207-215. John Willey and Sons, New York.

Guendouz A, Guessoum S, Maamri K, Benidir M and Hafsi M 2012 Canopy temperature efficiency as indicators for drought tolerance in durum wheat (Triticum durum Desf.) in semi arid conditions. J. Agric. Sustainability 1(1): 23-38.

$\mathrm{Hu} \mathrm{H}$ and Rajaram S 1994 Differential responses of bread wheat characters to high temperature. Euphytica 72: $197-$ 203.

Khanna-Chopra R and Viswanathan C 1999 Evaluation of heat stress tolerance in irrigated environment of $T$. aestivum and related species. I. Stability in yield and yield components. Euphytica 106: 169-180.

Koc M, Barutcular C and Tiryakioglu M 2008 Possible heat tolerant wheat cultivar improvement through the use of flag leaf gas exchange traits in a Mediterranean environment. J. Sci. Food and Agric. 88(9): 1638-1647.

Kohli MM, Mann CE and Rajaram S 1991 Global status and recent progress in breeding wheat for the warmer areas. $p p$ 96-112, In: Wheat for non--traditional warm areas. Saunders DA (ed.). , Mexico, D F, CIMMYT.

Mann CE 1994 Early vigor in wheat: A useful character for heat tolerance selection. In: Saunders DA and Hettel HP (eds.), Proc. Int. Congr. on Wheat in heatstressed environments: Irrigated, dry areas and rice-fanning systems. Mexico, DF, CIMMYT. 
Modarresi M, Mohammadi V, Zali A and Mardi M 2010 Response of wheat yield and yield related traits to high temperature. Cereal Res. Comm. 38(1): 23-31.

Ortiz R, Sayre KD, Govaerts B, Gupta R, Subbarao GV, Ban T, Hodson D, Dixon $\mathrm{JM}$, Monasterio JIO and Reynolds $\mathrm{M}$ 2008 Climate change: can wheat beat the heat? Agric. Ecosystems and Environment 126: 46-58.

Paknejad F, Nasri M, Tohidi Moghadam HR, Zahedi H and Alahmadi MJ 2007 Effect of drought stress on chlorophyll fluorescence parameters, chlorophyll content and grain yield of cultivars. J. Biol. Sci. 7(6): 841-847.

Rahman MM 1996 Screening technique for canopy temperature and leaf chlorophyll content in wheat. Bangladesh J. Pl. Genet. 9(1 \& 2): 1-6.

Rahman MM 2009 Genetic variation in the duration of growth stages and their relationship with yield and yield components in spring wheat under two sowing environments. MS Thesis, Dept. of Genetics and Plant Breeding, Bangladesh Agric. Univ. Mymensingh-2200, Bangladesh.

Rasal PN, Gavhane VN, Kusalkar DV, Gosavi AB and Shirpurkar GN 2006 Effect of high temperature stress on heat susceptibility index and thermal requirement of bread wheat (Triticum aestivum L.) genotypes. Res. Crops 7 (3): 811-813.

Rawson HM 1988 Effect of high temperatures on the development and yield of wheat and practices to reduce deleterious effects. pp 44-62. In: Klatt AR (ed.). Wheat Production Constraints in Tropical Environments. Mexico, D.F.: CIMMYT.

Rees D, Sayre K, Acevedo E, Sanchez TN, Lu Z, Zeiger E and Limon L 1993 Canopy temperatures of wheat: Relationship with yield and potential as a technique for early generation selection.
Wheat Special Report 10, Mexico, D F, CIMMYT.

Reynolds MP, Balota M, Delgado MIB, Amani I and Fischer RA 1992 Physiological and morphological traits associated with spring wheat yield under hot, irrigated conditions. Aust. J. Plant Physiol. 21: 717-730.

Reynolds MP, Nagarajan S, Razzaque MA and Ageeb OAA 2001 Heat Tolerance In: Reynolds MP, Ortiz-Monasterio JI and McNab A (eds.) Application of Physiology in Wheat Breeding, Mexico, D.F.: CIMMYT, 125 p.

Reynolds MP, Nagrajan S, Razzaque MA and Ageeb OAA 1997 Using Canopy Temperature Depression to select for yield potential of wheat in heat-stressed environments. Wheat Program Special Report. 42. Mexico, D F, CIMMYT.

Samad MA, Rahman MM, Amin MR, Sarker ZI and Islam M 1994 Selecting traits for breeding Heat-tolerant wheat varieties. In: (Saunders DA and Hettel HP Eds.), Proc. Int. Congr. on Wheat in heat stressed environments: Irrigated, dry areas and rice-wheat farming systems. pp. 379-81. Mexico, DF, CIMMYT.

Saunders DA 1988 Crop management research: Summary of results, 1983-88. Monograph No. 5: Wheat Research Center, Dinajpur, Bangladesh.

Sharma A, Rawat RS, Verma JS and Jaiswal JP 2013 Correlation and heat susceptibility index analysis for terminal heat tolerance in bread wheat. J Central European Agric, 14(2): 57-66.

Sharma RC, Tiwary AK and Ortiz-Ferrara G 2008 Reduction in kernel weight as a potential indirect selection criterion for wheat grain yield under terminal heat stress. P1. Breed. 127: 241-248.

Singh K, Sharma SN and Sharma Y 2011 Effect of high temperature on yield attributing traits in bread wheat. Bangladesh J. Agril. Res. 36(3): 415-426.

Singh RK and Chaudhury BD 1985 Biometrical methods in quantitative genetic 
analysis (Revised Ed.), pp 5-6. Kalyani publisher, Ludhiana, India.

Steel RGD and Torrie JH 1960 Principles and procedures of statistics. Mc-Graw Hill Book Co., Inc. New York.

Talebi R 2011 Evaluation of Chlorophyll Content and Canopy Temperature As Indicators For Drought Tolerance In Durum Wheat (Triticum durum Desf.). Aust. J. Basic and Applied Sci. 5(11): 1457-1462.

Wheeler TR, Hong TD, Ellis RH, Batts GR, Morison JIL and Hadley P 1996 The duration and rate of grain growth, and harvest index, of wheat (Triticum aestivum L.) in response to temperature and $\mathrm{C0}_{2}$. J. Exp. Bot. 47(298): 623-630.

Xiyong C, Qixin S and Zheng SC 2000 Performance and evaluation of spring wheat heat tolerance. J. China Agric. Univ. 5(1): 43-49.

Zahedi M and Jenner CF 2003 Analysis of effects in wheat of high temperature on grain filling attributes estimated from mathematical models of grain filling. J. Agric. Sci. 141: 203-212. 